\title{
DEATH FROM CEREBRAL AIR EMBOLISM DURING THORACOSCOPY
}

\author{
BY \\ SURGEON LIEUTENANT P. P. PHILIP, R.N. \\ From the Thoracic Surgical Unit, Royal Naval Hospital, Chatham
}

Many cases of patients who have died from air embolism during surgical operations have been reported, and in some of these the mode of death has suggested a cerebral lesion, but in very few have the brains been carefully examined at necropsy. As a result there is at present a state of uncertainty as regards the nature and pathology of cerebral air embolism.

Bohorfoush (1943) described finding "a larger than usual number of bubbles" in the veins on the surface of the brain of a negro who died following an artificial pneumothorax refill. Cory (1944) reported a case of death following bronchoscopy in which ophthalmoscopic examination performed just before death showed streams of air bubbles passing through the retinal vessels. There could be little doubt that cerebral air embolism was the cause of death in this case, yet no mention is made of post-mortem findings in the vessels of the brain. Ward (1922), in a case of death following urethroscopy, found bubbles of gas in all the chambers of the heart, the aorta, the common iliac arteries, circle of Willis, pulmonary veins, spermatic veins, and cerebral veins. Doyle and Frodsham (1949 described a case of death from air embolism following blood transfusion. They dismissed the fact that a few air bubbles were found in the cerebral veins, and stated that this is not an unusual finding. Several such statements suggesting that air bubbles are frequently found in cerebral veins at necropsy could be quoted. However, the position appears to be that normally there is no gas or air in blood vessels; air may be found in veins in bodies which are decomposed, particularly in hot climates, also in certain pathological states such as gas gangrene, air embolism, or "the bends." In addition if the skull-cap has been avulsed at necropsy in such a way that small tears have been produced in the venous sinuses, bubbles of air may sometimes be seen in these sinuses and also in the cerebral veins.
The following case of sudden death occurring during thoracoscopy and division of adhesions is of interest, as no reference to a similar occurrence has been noted in the literature, and evidence of cerebral air embolism was demonstrated at necropsy.

\section{CASE History}

The patient, a naval pensioner aged 43 years, first came under treatment for pulmonary tuberculosis in May, 1948, after a routine radiograph of the chest. This showed infiltration and cavitation in both upper zones. Sputum was positive for tubercle bacilli. The patient stated that his general health had always been very good. A general examination of the cardiovascular and central nervous systems revealed no abnormalities. After preliminary treatment by bed rest, a left artificial pneumothorax was induced on November 11, 1948. Apical adhesions were present, and so adhesion section was considered advisable. At no time was the left artificial pneumothorax under positive pressure. The last refill, given before thoracoscopy, was on January 5, 1949. Preliminary pressures were $-10-5 \mathrm{~cm}$. of water, and after a $600 \mathrm{ml}$. refill, $-2-0 \mathrm{~cm}$. of water. On January 7,1949 , a thoracoscopy was performed under local anaesthesia using $0.02 \%$ amethocaine hydrochloride. The usual routine pre-operative drugs had been given: omnopon, gr. $1 / 3$; scopolamine, gr. $1 / 150$, one and a half hours before, and a further gr. 1/3 of omnopon immediately before, operation. Throughout the operation the patient was lying on his right side with his head supported by one pillow.

The adhesions consisted of five fine apical strands, easily divisible. Two of these adhesions had been cauterized and the third was in the process of being divided, when the patient suddenly developed violent generalized clonic convulsions involving the trunk and limbs, became pulseless, and stopped breathing. Efforts at resuscitation were started at once. The head of the table was lowered and the patient given artificial respiration with oxygen; coramine was injected intravenously and adrenaline injected into the left ventricle. These efforts were of no avail. During the operation there was no bleeding and nothing to suggest that the adhesion which was being divided was unduly vascular. There were no warning signs ; indeed death was clinically instantaneous. 
It was assumed that cerebral air embolism was the cause of death, and that the air had entered a vein which had been partially opened by the cautery, or by one of the two cannulae which had been passed through the chest wall. It is emphasized that during thoracoscopy there is some communication between the pleural space and the atmospheric air throughout operation, and thus it is certain that the air in the pneumothorax space was not under positive pressure at any time during this operation.

Necropsy.-In view of the likelihood of cerebral air embolism being the cause of death, care was exercised in the examination of the brain, and facilities for photography were available. The necropsy was performed 48 hours after death. The weather was cold, and no putrefaction had taken place. The skull-cap was carefully removed and large bubbles of air were seen beneath the dura in the superior sagittal sinus. When the dura had been opened, numerous bubbles were seen in the cortical veins, mostly confined to the left side although a few were present in the veins on the right side close to their termination in the superior sagittal sinus. The brain was gently removed from the cranium and large bubbles of air were seen, completely filling the basilar artery and extending along the arteries forming the circle of Willis.

A well-marked patent foramen ovale was found of sufficient size to admit the tips of two fingers. The aorta contained frothy blood, but none was seen in the auricles or ventricles. No other abnormalities were noticed in the heart.

About a cupful of bloodstained fluid was present in the left pleural cavity. The adhesion which had been partially divided was covered with a small amount of blood clot, but although this adhesion and the remains of the divided adhesions were examined with care, no vessels were seen within them and no obvious portal of entry for air was apparent in the left pleural cavity. There was no evidence of trauma to the left lung or pleural cavity. The retinal vessels were not examined.

It was concluded that the cause of death was cerebral air embolism resulting from the entrance of air into a vein during division of adhesions.

\section{AIr Embolism}

There are various different types of air embolism. Air can enter the inferior or the superior vena cava from the systemic veins and can kill the patient by producing air locks in the right ventricle, provided large enough amounts are present. The air gets churned up with blood in the right ventricle to form a froth, and, because of its compressibility, this interferes with the expulsion of blood from the ventricle. With smaller amounts of air, insufficient to kill the patient in this way, bubbles will be carried on through the pulmonary arteries and lead to embolism of the smaller arteries of the lung. Such embolism is not harmful on account of the excellent collateral circulation present. According to the work of Catchpole and Gersh (1947) on decompression sickness, air emboli never reach the capillary bed, and observations by Chase (1947) on the mesenteric vessels of living animals confirm that gas bubbles introduced into the arterial circulation never penetrate beyond arterioles of about $40 \mu$ in diameter.

Air can enter one of the pulmonary veins at the hilum, probably at an operation such as pneumonectomy, and in this case it passes into the left auricle and on into the left ventricle to reach the systemic arteries, for example the cerebral arteries, producing cerebral air embolism. But although thoracic surgeons often inadvertently open the pulmonary veins, air never, in fact, seems to go into them. The reason is that the pressure in the veins is higher than in the vena cava and so much blood pours out that no air goes in ; consequently, although this type of air embolism is theoretically possible, it is unlikely to occur under normal circumstances. However, Mason (1936) described a case of death from cerebral air embolism, proved at necropsy, which occurred during lobectomy. In this case the upper lobe had been torn near the hilus, and while this was being repaired air was suddenly aspirated into a pulmonary vein. This aspiration occurred during a mishap to the anaesthetic apparatus, causing a sudden fall of the intrapulmonary air pressure.

Moore and Braselton (1940) performed many experiments with cats, in which they injected relatively small amounts of air into the pulmonary veins. In each of 30 experiments the cats died, apparently from air embolism of the coronary arteries; the emboli could be seen entering these arteries during the experiments and causing air locks in them.

Air emboli may reach the pulmonary veins during thoracic operations by way of the bronchial veins. Bronchial veins are said to drain both peripherally and centrally and to some extent directly into the pulmonary veins. Veins from the larger bronchi and from the lymphatic glands are also said to drain directly into the pulmonary veins rather than the bronchial veins, which usually terminate in the vena azygos major on the right and the superior hemiazygos on the left (Harris and Lewis, 1940).

Air which enters a systemic vein passes to the right auricle, and from there can get into the left auricle through a patent foramen ovale. After this it is distributed to the points in the systemic circulation and notably to the arteries in the base of 
the brain. This is the so-called "paradoxical embolism." Cohnheim in his Lectures on General Pathology (1882) described a case of paradoxical embolism occurring in a woman who had extensive venous thrombosis in the veins of the leg and who died from embolism of the middle cerebral artery. At necropsy a large foramen ovale was

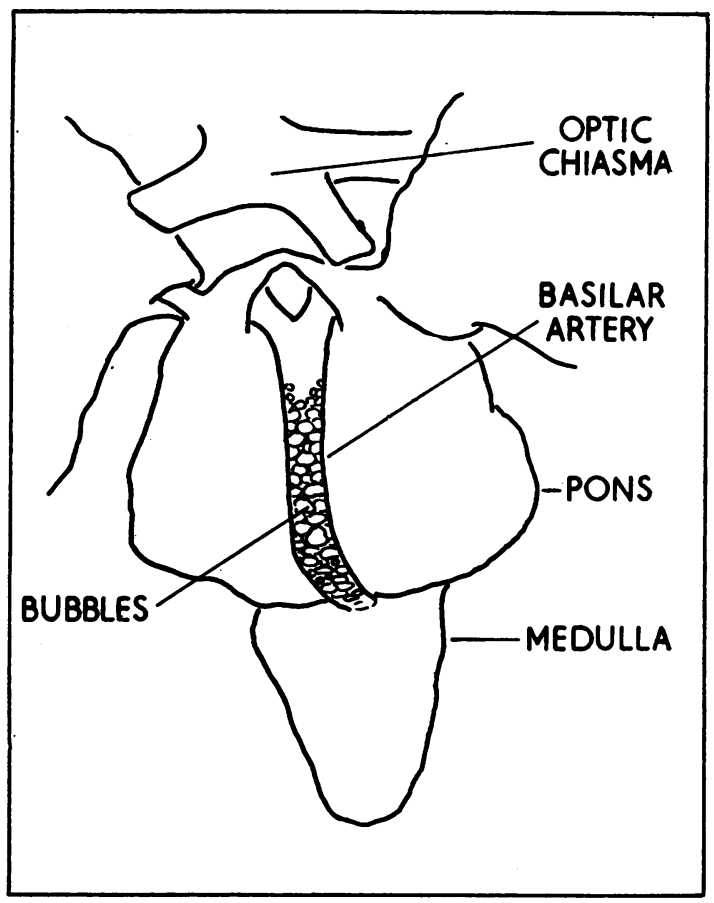

FIG. 1.-Diagram of pons and medulla, showing basilar artery filled with air emboli.

discovered which would admit three fingers. No other possible origin of the embolus could be found in the heart or aorta. The only objection to the theory of paradoxical emboli is that normally the flow through a patent foramen ovale is from left to right. The mean pressure in the left auricle has been measured in cases of atral septal defect, and is stated by Cournand and others (1947) to be higher than that in the right auricle, and therefore passage of air emboli from the right auricle to the left auricle would not be favoured.

Air can enter the paravertebral plexus of veins. This can occur if air gets into one of the veins of the chest wall or any vein directly or indirectly connected with the paravertebral plexus. In this event some of the air bubbles tend to lodge in the veins on the surface of the brain in those patients who die. If air is found in the cerebral veins or venous sinuses and is of pathological significance, the only possible way it can have got there is from the paravertebral plexus.

Harris (1941), in a description of the clinical anatomy of the paravertebral plexus, pointed out that the detailed anatomy of these veins of the spine was well known and described as early as 1664 by Willis, and in 1823 a very accurate description was published by Boeck, of Leipzig. These well established facts about the plexus have been "re-discovered" from time to time and used to explain the passage of emboli to the brain, and to account for such diverse phenomena as the tendency of carcinoma of the prostate to spread to the bodies of the lumbar vertebrae, of carcinoma of the breast to metastasize to the spine, or of chronic pulmonary suppuration to engender cerebral abscess. Purdon-Martin (1941) described attacks of sagittal sinus thrombosis with convulsions and paresis following childbirth in which he postulated the migration of fragments of clot from the pelvic veins to the cerebral veins through this venous system. Batson (1940) gave a detailed account of the paravertebral veins and also a description of some interesting injection experiments on cadavers which demonstrated the free anastomoses which exist between this venous system and the veins of the pelvis, the intercostal veins, the azygos veins, and the intracranial sinuses. He also performed injection experiments on living monkeys in which opaque material was injected into the dorsal vein of the penis and the path taken by the material was followed radiologically. He showed that under normal conditions the venous flow was along the pelvic veins into the inferior vena cava, but under the simulated physiological conditions of coughing and straining (produced by a tight abdominal binder) the flow was into the vertebral plexus, vessels of the thoracic spine, and lower intercostal vessels. This showed in effect that during coughing, compression of the chest, and straining, blood is not only prevented from entering the chest, but is actually squeezed out of the intra-abdominal veins into the paravertebral venous system. It would appear that this venous system acts as a "safety valve" in the systemic venous circulation during acts of coughing and straining.

Batson also discovered, by injection of opaque material into the veins of the breast in a cadaver, that the solution found its way to the intercostal veins, transverse cranial sinuses, and superior longitudinal sinus through this plexus. 
Collis (1944) performed similar experiments, and demonstrated that the opaque solution, when injected into an intercostal vein, reached the veins of the cranial cavity by way of the paravertebral plexus, indicating the probable route taken by septic emboli producing cerebral abscess in cases of chronic thoracic suppuration.

The paravertebral venous system is composed of thin-walled vessels, indicating that the blood contained in them is under low pressure. They possess no effective valves and thus a reversal of flow can occur in them subject to a rise or fall of venous pressure in the thorax or abdomen. The system has considerable volume, and is capable of storing large quantities of blood. The veins are difficult to identify when empty, as in dissection of the cadaver, but may often be clearly seen in the living subject at operations. The plexus has numerous connexions with the veins in the spinal canal and those within the vertebrae themselves. In lateral radiographic views of the spine in young children, notches may be seen in the anterior surfaces of the vertebral bodies for the transmission of branches of the anterior external vertebral plexus which runs longitudinally in front of the vertebral bodies. These branches pass into the bodies of the vertebrae and communicate therein with the basivertebral veins, which form an anas-

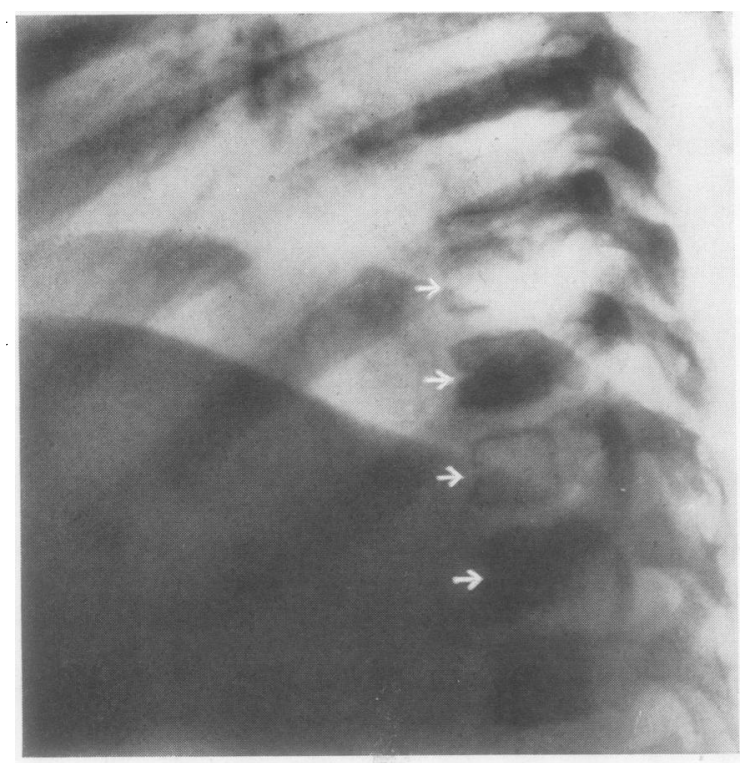

FIG. 2.-Lateral film of spipe in a child of eight months showing the notches in the anterior surfaces of the vertebral bodies for the branches of the anterior external vertebral plexus which pass into the vertebral bodies and communicate with the basivertebral veins. tomosis with the internal vertebral venous plexuses lying within the vertebral canal. These communicating branches of the anterior external plexus are vessels of considerable size in young subjects, as can be appreciated by the size of the notches in the vertebrae shown in the accompanying lateral radiograph of the spine in a child of eight months.

The paravertebral venous plexus communicates directly with the intercostal veins through their dorso-spinal branches. Also with the veins of the breast and the azygos veins. Through the azygos veins there exist free communications with the posterior bronchial veins and the veins of the parietal pleura. Numerous anastomoses occur between the paravertebral system and the pelvic viscera. Occasional communications with the renal veins are present.

Bohorfoush (1943) has suggested that in cases of air embolism resulting from a pneumothorax the bubbles could pass from the intercostal vein through the azygos system to the superior vena cava, and, if the patient were erect or partly so, could float upstream in the internal jugular vein to the cerebral sinuses. This is of course theoretically possible, but is unlikely to have occurred in the particular case described here as the patient was lying horizontally throughout operation.

The interest of the case under discussion is that it combines two different types of air embolism, for emboli were found in the basal arteries of the brain as well as in the cortical veins and superior sagittal sinus. It is suggested that the bubbles in the basilar artery and circle of Willis were paradoxical emboli reaching the right auricle via the systemic veins draining the chest wall. The bubbles found in the cortical veins and superior sagittal sinus must have taken another route, for it is unlikely that the air emboli found in the basal arteries could have negotiated the cerebral capillaries and passed through into the veins. The emboli must have arrived in these vessels via the paravertebral plexus, probably originating in an intercostal vein and passing into the plexus by way of the dorso-spinal branch.

\section{Behaviour of Air EMboli}

It may not be generally understood why it is that a bubble of air in the circulation will act as an embolus. If blood will pass through the smaller vessels, why cannot air? The obstruction produced by air in the circulation is apparently due to the fact that it is present in bubbles having a highly resistant liquid film. It has been shown by Wilson and Reis (1923) that with certain colloidal solutions the surface films of the foam behave as gel- 


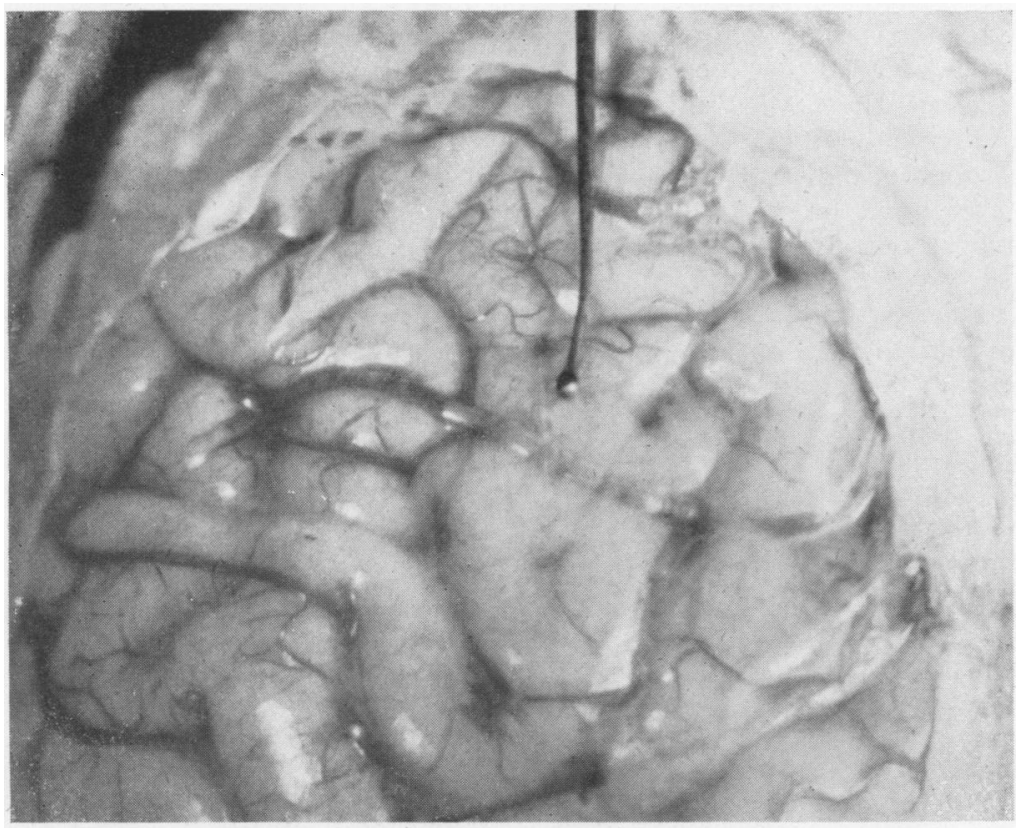

FIG. 3.-A view of the left cerebral hemisphere showing air emboli in the cortical veins. The superior sagittal sinus is to the right of picture, and a collection of emboli can be seen in a cortical vein at its termination in the sinus in the upper part of the picture.

like plastic solids ruther than as viscous liquids, and as a result the superficial viscosity may be more than a thousand times that of water. It is well known that protein films on water possess great viscosity and elasticity. The surface film of an air bubble in blood has similar properties and such a bubble is extremely resistant to rupture.

Air emboli do not behave in the same way as other emboli: a bubble of air in a tube does not necessarily impact at a bifurcation nor does it necessarily continue to pass onwards until it reaches the point at which the vessel can no longer accommodate it; it is influenced by kinks in the vessel and also by the fact that it may have reached the highest point of a bend. Considering the tortuosity of the cerebral vessels, they are ideal theoretically for catching up bubbles of air and so causing air locks in the cerebral circulation. Air emboli are greatly influenced by posture, and bubbles of air in the circulation will tend to move to the highest point in the blood stream. An example of this fact is apparent when air is accidentally introduced into the circulation during an artificial pneumothorax refill. The patient is usually lying horizontally during this procedure, and signs of those cerebral lesions which may arise as a result of this catastrophe often do not begin to appear until the patient assumes the erect posture.

\section{Discussion}

Air embolism of the brain usually kills the patient very quickly-that is, quickly in relation to the time which elapses between the first physical sign and death. This is generally true in fatal cases of what has been called "pleural shock" but is now known to be air embolism. In the case under discussion, death was clinically instantaneous and presumably coincided with the entrance of emboli into the basilar artery and circle of Willis. With the abrupt cessation of circulation there was little chance of the bubbles becoming propelled further onwards and dispersed along the smaller branches of these vessels and thus disappearing from view. The emboli were therefore still present in the large vessels and could be seen clearly at necropsy. It would seem feasible to suggest that in cases where death is not quite so sudden, either because the emboli are smaller and less numerous or because the bubbles of air enter the circulation more slowly, there is more time for the cerebral circulation to propel the emboli further onwards into the smaller branches where the fatal damage is done out of sight of the pathologist at necropsy. This may partly explain why it is often difficult to demonstrate air emboli in the cerebral vessels at post-mortem examination in cases supposedly dying as a result of this complication. It is suggested that, when death occurs during surgical procedures and cerebral air embolism is suspected, the necropsy should be performed as soon as possible, before putrefaction begins. Great care should be exercised in removing the skull-cap and also in taking the brain from the cranial cavity in order not to tear the dura or cerebral veins and thus leave the pathologist in doubt as to whether bubbles found in vessels are artefacts produced after death or air emboli causing death. It is also possible that air may gain entrance to the cerebral veins and sinuses during post-mortem examination through cutting the veins in the neck when the tongue and pharynx are 
removed, if this is done before the brain is examined. It is recommended that, where the possibility of cerebral air embolism exists, the brain should always be examined before removing these structures, as was done at the necropsy on the case I have described. If bubbles are found in the cerebral veins or sinuses at necropsy and are of pathological significance, then it should be assumed that those bubbles have reached there by way of the paravertebral plexus. Veins near the site of operation, having communication with this plexus, should be scrutinized carefully for contained air bubbles, and an attempt should be made if possible to find exactly where the air gained entrance to the circulation. For instance, in cases of death during surgical procedures performed on the chest, such as an artificial pneumothorax refill, the intercostal veins, the azygos veins, and the bronchial veins should be carefully examined.

\section{SUMMARY}

A case of sudden death from cerebral air embolism during thoracoscopy and division of adhesions is described. Post-mortem examination revealed large bubbles of air in the superior sagittal sinus and cortical veins, and in the basilar artery and circle of Willis.

The passage of emboli is discussed anatomically.
I wish to express my thanks to Mr. N. R. Barrett, consultant in thoracic surgery to the Royal Navy, and to Professor H. A. Harris, of Cambridge University, for help and advice, and to Surgeon Rear-Admiral K. A. I. Mackenzie for permission to publish this case.

\section{REFERENCES}

Batson, O. V. (1940). Ann. Surg., 112, 138.

Bock, A. C. (1823). Darstellung der Venen. Leipzig. Bohorfoush, J. G. (1943). Amer. Rev. Tuberc., 47, 263. Catchpole, H. R., and Gersh, I. (1947). Physiol. Rev., 27,360 .

Chase, W. H. (1934). Surg. Gynec. Obstet., 59, 569.

Cohnheim, J. (1889). Lectures on General Pathology, Sydenham Series, 1. London.

Collis, J. L. (1944). J. thorac. Surg., 13, 445.

Cory, R. A. S. (1944). Ibid., 13, 30.

Cournand, A., Motley, H. L., Himmelstein, A., Dresdale, D., and Baldwin, J. (1947). Amer. J. Physiol., 150, 267.

Doyle, G. B., and Frodsham, P. (1949). Lancet, 1, 735.

Harris, H. A. (1941). Brain, 64, 291.

- and Lewis, I. (1940). J. thorac. Surg., 9, 666.

Mason, G. A. (1936). Lancet, 4, 1047.

Moore, R. M., and Braselton, C. W. (1940). Ann. Surg., $112,212$.

Purdon-Martin, J. (1941). Brit. med. J., 2, 537.

Ward, R. O. (1922). Proc. R. Soc. Med., 16, 54.

Wilson, R. E., and Reis, E. D. (1923). Surface Films as Plastic Solids. Pp. 145-173. 